Anesthesiology 1998; 88: 327-33.

6 Koch CG, Khandwala F, Li L, Estafanous FG, Loop FD,

Blackstone EH. Persistent effect of red cell transfusion on health-related quality of life after cardiac surgery.

Ann Thorac Surg 2006; 82: 13-20.

\section{Perioperative blood conservation strategies: weighing the medical evidence - II}

To the Editor:

In their recent editorial ${ }^{1}$ on the subject of perioperative blood conservation, Drs. Karkouti and McCluskey present a perspective which is at variance with some of the tenets of evidence-based medicine. ${ }^{1}$ As Drs. Goodenough and Shander have stated, "Blood management is most successful when multidisciplinary, proactive programs are in place so that these strategies can be individualized to specific patients". Evidence-based transfusion practice views a patient's blood as a valuable and a unique resource that should be managed appropriately. Allogeneic blood should only be used when there is evidence for potential benefit, when there are no safer alternatives, when a quality product is available, and after the risks have been appropriately considered. Patient-focused blood management is a multidisciplinary and a multimodal medical practice that starts with an accurate diagnosis of a patient's blood-related issues, through to the development of short and long-term management plans, with appropriate monitoring of outcomes.

On the basis of their editorial, ${ }^{1}$ it is uncertain how Drs. Karkouti and McCluskey would view the role of randomized controlled trials (RCTs) in confirming: the importance of pretransfusion compatibility testing; the avoidance of transfusion-transmitted viral diseases; the benefits of preoperative assessment for surgical bleeding and thromboembolic risk; and the benefits of correcting preoperative, reversible anemia in elective surgical cases. The recently published Austrian benchmark study on blood use in elective surgery ${ }^{3}$ demonstrates a high degree of interhospital variability in red cell transfusion rates for selected surgical procedures (e.g., for elective hip arthroplasty between $16 \%-85 \%$ of patients are transfused) that would be difficult to reconcile through a randomized controlled trial.

There is strong observational evidence indicating that perioperative, allogeneic red cell transfusion is an independent risk factor for increased morbidity and mortality. When concerns arise regarding the benefit and safety of a clinical intervention, especially where established alternatives are available, the precautionary principle should be applied. In transfusion medicine, this would mandate a non-transfusion default position. In this respect, it is ironic that blood transfusion is viewed differently from other therapies. Jehovah's Witness patients can safely undergo most surgical procedures, without allogeneic transfusion, with better clinical outcomes, especially in cardiac surgery, when compared with control matched, transfused patients. ${ }^{4}$

Drs. Karkouti and McCluskey imply that a numerical analysis frequentist approach to evidence (i.e., RCTs) is the only valid methodology to establishing causation where there is correlation, and that a sound understanding of pathophysiology and process decision analysis (Bayesian analysis) are of lesser validity. However, we should note that Dr. Archie Cochrane, of Cochrane Database fame, developed registries of observational data as the foundation for his epidemiological research. ${ }^{5}$ Even more importantly, Dr. Austin Bradford Hill proposed criteria for supporting causation from correlation when RCTs could not, or would never be done (e.g., smoking causing lung cancer). ${ }^{6}$ All medical therapies have associated risks and, recognizing that evidence for benefit is commonly lacking, RCTs have an important, but not exclusive, role in contributing to the establishment of best medical evidence. The recently identified safety concerns surrounding aprotinin and erythroid stimulating agents are concerning, and clearly need to be taken into account in assessing risk/benefit in modern perioperative transfusion medicine. Rather than being "elephants", aprotinin and erythroid stimulating agents are only two recently advocated pharmacological aspects of blood management. At the core of patient blood management is scientifically based clinical medicine dating back over 100 years.

The authors state that, for "the clinician", "The basic tenet of clinical medicine is, first, do no harm". They apply this dictum to two possible transfusion alternatives, but they do not acknowledge the vast observational and limited RCT data incriminating allogeneic transfusion as an independent risk factor for poorer clinical outcomes. The "experts" in patient blood management are not advocating the use of every blood conservation strategy in every patient, and are not striving for universal, bloodless medicine. However, the experts are advocating the practice of good clinical medicine, to minimize allogeneic blood transfusion and to conserve an altruistically donated resource that should be used for the benefit of the greatest number of patients. As for throwing down the "gauntlet", the patient blood management "gauntlet" has been at the authors' feet for many years. 
James P. Isbister BSC(Med) MB BS FRACP FRCPA

Royal North Shore Hospital of Sydney, St Leonards, NSW, Australia

E-mail: jisbiste@med.usyd.edu.au

Disclosures: Chair, Advisory Committee and Board Member Australian Red Cross Blood Service, Chair, Haemostasis Register of rVIIa use in Australia and New Zealand

Occasional consultant to CSL Bioplasma Ltd, Australia, Novo Nordisk Ltd, Australia and Amgen Australia. Accepted for publication December 17, 2007.

\section{References}

1 Karkouti K, McCluskey SA. Perioperative blood conservation - The experts, the elephants, the clinicians, and the gauntlet (Editorial). Can J Anesth 2007; 54: 861-7.

2 Goodnough LT, Shander A. Blood management. Arch Pathol Lab Med 2007; 131: 695-701.

3 Gombotz H, Rehak P, Shander A, Hofmann A. Blood use in elective surgery: the Austrian benchmark study. Transfusion 2007; 47: 1468-80.

4 Reyes G, Nuche JM, Sarraj A, et al. Bloodless cardiac surgery in Jehovah's Witnesses: outcomes compared with a control group (Spanish). Rev Esp Cardiol 2007; 60: 727-31.

5 Cochrane AL. The detection of pulmonary tuberculosis in a community. Br Med Bull 1954; 10: 91-5.

6 Hill $A B$. The environment and disease: association or causation? Proc R Soc Med 1965; 58: 295-300.

\section{Reply:}

We appreciate the opportunity to respond to the letters by Gill et al. and Isbister regarding our editorial on perioperative blood conservation ${ }^{1}$ and begin by clarifying our perspective on the issue. Since we do not know if any of the available blood conservation modalities has a better risk-benefit profile than allogeneic blood, we should limit their use until this information becomes available. Until then, if we are considering using any of these modalities, we should fully inform our patients of this knowledge gap. To be clear, we are not proposing that only randomized controlled clinical trials be used to define the risk-benefit profiles of blood conservation modalities. On the contrary, we strongly believe that the totality of evidence, including observational studies, should be used for this purpose. It is imperative, however, that all studies, be they randomized or observational, be critically appraised before we draw any conclusions from them.
While it is true that allogeneic blood is a scarce, expensive resource, currently the main argument for perioperative blood conservation seems to be that allogeneic blood is such a strong, independent risk factor for postoperative morbidity and mortality that we should do all that we can to avoid even one or two unit transfusions. This argument is primarily supported by observational studies that have found red blood cell (RBC) transfusion to be a strong risk factor for adverse outcomes in cardiac surgery. ${ }^{2,3}$ Whether such studies can determine the independent relationship between $R B C$ transfusion and adverse outcomes, by adequately adjusting for important confounders, however, is questionable. ${ }^{4}$ As is elegantly explained by Deans et al., ${ }^{5}$ the use of RBC transfusions is titrated, based not only on the nature and severity of patients' underlying disease, but also on their overall health status. Existing studies, however, did not measure and, consequently, could not adjust for patients' overall health status. ${ }^{2,3}$ Moreover, blood transfusion is often the outcome of perioperative adverse events - either directly, when the adverse event leads to blood transfusion, or indirectly, when the adverse event leads to prolonged hospitalization which, in turn, leads to hospital-acquired anemia. Risk-adjustment, in these studies, does not account for this confounding relationship.

To demonstrate, let us assume that two identical patients (with respect to co-morbidities, intraoperative course, and laboratory values) present with a hematocrit of $23 \%$ in the immediate post-cardiopulmonary bypass (CPB) period. Patient-1 has an uneventful postoperative course, but Patient-2 develops sustained ST-segment depression, consistent with myocardial ischemia. Given that myocardial ischemia in the setting of low hemoglobin concentration is an indication for RBC transfusion, ${ }^{6}$ and post-CPB ST-segment depression is associated with adverse cardiac outcomes, ${ }^{7}$ Patient-2 is more likely to receive a $R B C$ blood transfusion, to suffer a myocardial infarction, and to die. Thus, unless the incidence, duration, and temporal pattern of perioperative STsegment depression are measured and properly adjusted for by multivariable analysis, an observational study comparing patients who did, and who did not, receive $R B C$ transfusions will find a spurious association between RBC transfusion and postoperative mortality. Unfortunately, this and many other important bealthstatus and adverse outcome confounders (such as measures of perioperative tissue bypoxemia, hemodynamic instability, and renal dysfunction) were not modified in these retrospective observational studies assessing the effects of RBC transfusions.

Thus, critical appraisal of these studies reveals that it is quite possible that the observed association between 
$R B C$ transfusion and adverse events is due to the effects of unmeasured confounders. It is also interesting to note that, in these studies, about five units is the RBC transfusion threshold beyond which risk is markedly increased. This is consistent with our previous work, ${ }^{8}$ and suggests that the risk attributed to $R B C$ transfusions may be due to massive blood loss that necessitates large-polume resuscitation, which leads to marked physiological perturbations including acid-base and electrolyte abnormalities, tissue edema, hemostasis impairment, and immune and inflammatory system modulation, all of which have been linked to end-organ ischemia and dysfunction. None of the existing blood conservation modalities has been shown to reduce the incidence of massive blood loss.

In conclusion, while we do not doubt the importance of multidisciplinary blood management programs aimed at reducing blood loss and at lowering the exposure to blood products, we believe that only modalities with proven, favourable risk-benefit profiles should be employed to achieve these objectives. Anything less and we may be providing fresh fodder for the old cliché, "the operation was a success, but the patient died." The validity of this concern is well illustrated by the premature termination of the blood conservation using antifibrinolytics: a randomized trial in a cardiac surgery population trial, apparently because it found that, while patients who received aprotinin had lower blood loss compared to those who received other antifibrinolytic drugs, their mortality rate was higher. ${ }^{A}$

Keyvan Karkouti MD FRCPC MSc

Stuart A. McCluskey PhD MD FRCPC

Toronto General Hospital, University of Toronto, Toronto, Canada

E-mail: keyvan.karkouti@uhn.on.ca

\section{References}

1 Karkouti K, McCluskey SA. Perioperative blood conservation - The experts, the elephants, the clinicians, and the gauntlet (Editorial). Can J Anesth 2007; 54: 861-7.

2 Koch CG, Li L, Duncan AI, et al. Morbidity and mortality risk associated with red blood cell and bloodcomponent transfusion in isolated coronary artery bypass grafting. Crit Care Med 2006; 34: 1608-16.

3 Murphy GJ, Reeves BC, Rogers CA, Rizvi SI, Culliford $L$, Angelini GD. Increased mortality, postoperative

A U.S. Food and Drug Administration. Early communication about ongoing safety review - aprotinin injection (marketed as Trasylol). Available from URL; http://www.fda.gov/cder/ drug/early_comm/aprotinin.htm (accessed December 6, 2007). morbidity, and cost after red blood cell transfusion in patients having cardiac surgery. Circulation 2007; 116: 2544-52.

4 Karkouti K, Wijeysundera DN, Yau TM, et al. Platelet transfusions are not associated with increased morbidity or mortality in cardiac surgery. Can J Anesth 2006; 53: 279-87.

5 Deans KJ, Minneci PC, Suffredini AF, et al. Randomization in clinical trials of titrated therapies: unintended consequences of using fixed treatment protocols. Crit Care Med 2007; 35: 1509-16.

6 Spabn DR. Strategies for transfusion therapy. Best Pract Res Clin Anaesthesiol 2004; 18: 661-73.

7 Smith RC, Leung JM, Mangano DT. Postoperative myocardial ischemia in patients undergoing coronary artery bypass graft surgery. S.P.I. Research Group. Anesthesiology 1991; 74: 464-73.

8 Karkouti K, Wijeysundera DN, Yau TM, et al. The independent association of massive blood loss with mortality in cardiac surgery. Transfusion 2004; 44: 1453-62.

\section{History of anesthesia; Mallampati revisited: 20 years on}

To the Editor:

It is more than 20 years since the publication of " $A$ clinical sign to predict difficult tracheal intubation using a laryngoscope: a prospective study" by Mallampati et al. ${ }^{1}$ Many anesthesiolgists who read this paper were left with the impression that a Mallampati class 3 or 4 airway has a high positive predictive value (PPV) for difficult intubation. This is not supported by any study other than the original report. ${ }^{1}$ More recent studies report dramatically lower PPV values. To appreciate how this situation evolved, it is necessary to examine Mallampati et al.'s original paper in the context of developments in the anesthesia literature over the past 20 years.

Mallampati et al.'s original paper described three classes of oropharyngeal view and a later modification, by Samsoon and Young, ${ }^{2}$ added a fourth class. This latter classification is now often referred to as the modified Mallampati classification. In 1985, however, Mallampati et al. were not using the Cormack \& Lehane $^{3}$ grading of laryngeal view, which had been published only the year before, and was not in widespread use at that time. Mallampati et al. developed their own system of four grades of laryngeal view as follows: grade 1 - glottis could be fully exposed; grade 2 - glottis could be partly exposed; grade 3 - glottis could not be exposed; grade 4 - glottis, including 